\title{
Intermediate energy ionization of helium by proton impact
}

\author{
E Edgü-Fry, C L Cocke, E Sidky, C D Lin and M Abdallah \\ Department of Physics, JR Macdonald Laboratory, Kansas State University, Manhattan, \\ KS 66506, USA \\ E-mail: erge@phys.ksu.edu
}

Received 27 February 2002, in final form 23 April 2002

Published 24 May 2002

Online at stacks.iop.org/JPhysB/35/2603

\begin{abstract}
We have investigated impact ionization of $\mathrm{He}$ by protons at energies between 20 and $100 \mathrm{keV}$. Momentum spectra of the ejected electrons were measured for experimentally determined vector impact parameters using cold target recoil ion momentum spectroscopy techniques. At the lowest impact energy, the electron momenta lie close to the saddle point and as the energy increases they slowly move towards the target centre. The measurements are compared with the results of a theoretical calculation carried out using a two-centre momentum space discretization method. Qualitative agreement with the experiment is seen, and systematic disagreements between experiment and theory are discussed.
\end{abstract}

\section{Introduction}

The collisional removal of an electron from a neutral target by a projectile whose velocity is smaller than or comparable to that of the active electron has been the subject of studies for more than half a century. In this low-velocity regime the dominant electron removal process is electron capture, a process that is rather well understood theoretically. On the other hand, the weaker process of direct electron ejection into the continuum has been the subject of considerable controversy. At the heart of the difficulty of dealing theoretically with this process lies the treatment of the low-energy electron continuum. Much discussion has appeared in the literature [1-16] concerning the role of 'saddle point' ionization and associated classical and quantal treatments of this process. Over the past few years, the experimental side of the issue has advanced considerably with the use of imaging techniques which have allowed the full momentum space distribution of the very low-energy ejected electrons to be measured for fixed values of the vector impact parameter [17-20]. For singly and doubly charged projectiles on atomic targets, these electron momentum distributions have been found to centre in velocity space around the location of the saddle point formed in the potential of the two receding heavy fragments, and to display marked structure which is attributable to the structure of the dominant participating molecular orbitals promoted into the continuum during the collision. 
The ionization of He by protons has been a frequent test case for the ionization process because of the simplicity of the target. Dörner et al [17] used the technique of cold target recoil ion momentum spectroscopy (COLTRIMS) in studying electron ejection in the protonhelium system for low impact energies $(5-15 \mathrm{keV})$ using the recoil momentum of the target $\mathrm{He}^{+}$fragment to select the collision plane and impact parameter. They found that the ejected electrons lie mainly in the scattering plane. Their longitudinal velocities were found to lie between those of the target and the projectile, while their transverse velocities in the scattering plane were typically below a few tenths of an atomic unit. The velocity distributions in the scattering plane showed a two-finger structure, one finger extending away from the direction of the projectile scattering and one toward it. The relative intensity of these two fingers was found to oscillate with changing energy projectile energy. This behaviour was first explained qualitatively by Ovchinnikov and Macek [3-5]. The origin of the oscillation was attributed to interference between $\sigma$ and $\pi$ amplitudes in the continuum, with a relative phase, which varied inversely with the projectile velocity. In this interpretation, the enabling first step for continuum electron production is the rotational coupling of the $2 \mathrm{p} \sigma$ and $2 \mathrm{p} \pi$ states in the $\mathrm{H}_{2}^{+}$-like transient molecule. The $\pi$-structure of this orbital is then promoted into the continuum where its characteristic nodal line along the internuclear axis is seen as an experimental minimum in the velocity-space images. However, precisely defining what these molecular states evolve into in the continuum and their interaction has been problematic. Ab initio calculations by Sidky and Lin $[9,10]$ reveal the $\pi$-structure in the continuum, but they show different energy dependence than Macek and Ovchinnikov's calculations [5], suggesting that the harmonic approximation for the saddle potential and the two-state expansion of the continuum, used in [5], might not be adequate. A more general explanation for the $\pi$-structure in the continuum has been proposed recently by Sidky et al [10], where they suggest that the minimum along the internuclear axis is essentially due to the shadow cast by the bound states on the continuum in velocity space. This $\pi$-structure has since been reported for several other collision systems [18-20] and seems to be a general characteristic of the velocity space distributions in the low-velocity regime as long as the projectile is not highly charged. Very different distributions are seen if the projectile is highly charged [22]. In this case, the saddle-point mechanism does not appear to be the dominant process for ionization in the energy region investigated.

At projectile velocities above about 3 au, the ionization process becomes perturbative in nature, with the electron momentum distributions centring on the target after the collision. The typical momenta of the continuum electrons are of a magnitude similar to that of the bound state electrons before ejection. Non-coincident electron spectra from this process have been heavily studied for decades and treatments by various forms of perturbation theory have found considerable success [23]. Imaging data for ionization of $\mathrm{He}$ by fast protons have been taken by several authors [24-27]. At these higher velocities it is no longer possible to identify a scattering plane unambiguously, because the momentum transfers between projectile and electron and between projectile and target nucleus are comparable, and because the use of classical trajectories becomes invalid in principle. Two major mechanisms for electron ejection have been identified, namely 'dipole-like' ionization involving momentum exchange between electron and target ion (recoil) and 'binary encounter' or 'two-body' ionization, involving momentum exchange between projectile and electron [24]. In the former process the projectile provides energy but not momentum, analogous to photoionization. Perturbation theory calculations, in particular the CDW incarnation [28,29], accounts well for all aspects of the data taken to date on the $\mathrm{p}-\mathrm{He}$ collision system.

In this paper we present the results of a COLTRIMS study of the ionization of He by protons between 20 and $100 \mathrm{keV}(v=0.8-2 \mathrm{au})$. This energy region bridges the gap between the non-perturbative 'saddle point' region and the perturbative region, and addresses the question 
of how the evolution from the low-energy picture to the high energy proceeds. In particular, we examine to what extent the structured behaviour observed by Dörner et al [17] is retained as the collision time decreases. We compare our results with those from a theoretical calculation recently developed to deal with ionization in one-electron collision systems by Sidky and Lin and show the successes and limitations of employing a model potential to apply this oneelectron theory to a two-electron collision system.

\section{Experimental method}

The experiments were conducted at the KSU-CRYEBIS facility. The ion beam, collimated to $1 \mathrm{~mm}$ in diameter, intersected at right angles a $3.5 \mathrm{~mm}$ wide supersonically cooled He gas jet with a density of about $10^{12}$ atoms $/ \mathrm{cm}^{3}$. The target was first cooled through thermal contact with a cold head cryopump at $60 \mathrm{~K}$ and supersonically cooled by passage through a $30 \mu \mathrm{m}$ diameter aperture. This produced a supersonic flow of the gas where most of thermal velocity was converted into a directional velocity in the direction of flow of the gas. The jet was skimmed by a conical skimmer of aperture diameter $0.5 \mathrm{~mm}$, keeping the cooler inner part of the jet. In the collision region the internal temperature of the gas was below $1 \mathrm{~K}$, corresponding to a momentum spread below 0.2 au. Both the recoil ions and electrons were directed by an external electric field of about $25 \mathrm{~V} \mathrm{~cm}^{-1}$ towards position sensitive channel plate detectors (PSCD). The electrons were detected after travelling through a short acceleration range $(23 \mathrm{~mm})$. A small negative bias voltage was applied to the first channel plate of this detector to repel background electrons. The recoils were accelerated in the opposite direction over a distance of $86 \mathrm{~mm}$ and subsequently traversed a field-free region of $305 \mathrm{~mm}$. The extraction field was shaped slightly so as to focus a parallel beam from the interaction region onto the recoil detector and was arranged also to provide first-order time focusing for the recoil ions. Both particles' times of arrival and positions were measured and the products were detected in time coincidence. Two components of both recoil-ion and electron momenta were reconstructed from position information from the respective detectors. The times-of-flight of the electrons was only a few nanoseconds whereas it was a few microseconds for recoil-ions. The spread in the time of flight of electrons was about $1 \mathrm{~ns}$, which made measuring the third component of the electron velocity impossible. Instead, the time signal of the electrons was used to give the start signal for a time-to-amplitude converter, which was stopped by the recoil-ion signal. This allowed us to measure the time of flight of the recoil ions and thus the third component of their momentum. From the position and time of flight, we derived the longitudinal and the transverse components of the final state momentum of both electrons and recoiling ions. The coordinate system used was the following: the projectile beam defined the $z$-axis, the gas jet defined the $y$-axis and $x$-axis was parallel to the electric field and perpendicular to the detector planes. Further detail on the experimental setup can be found in [20].

\section{Qualitative discussion of experimental results}

Figure 1 shows two-dimensional density plots of the electron velocity spectra projected onto the $y-z$ plane. The recoil momenta have been selected to lie in the negative $y$-direction, so that these spectra represent pictures of the electron spectra viewed looking 'down onto' the collision plane from above, where the collision plane is defined by the beam axis and the direction of the recoil momentum. As discussed previously [17], since the electron velocities lie approximately in the collision plane, this view provides the maximum information on the character of the electron velocity distributions. The units are $v_{e} / v_{p}$ where $v_{e}$ is the electron velocity and $v_{p}$ 

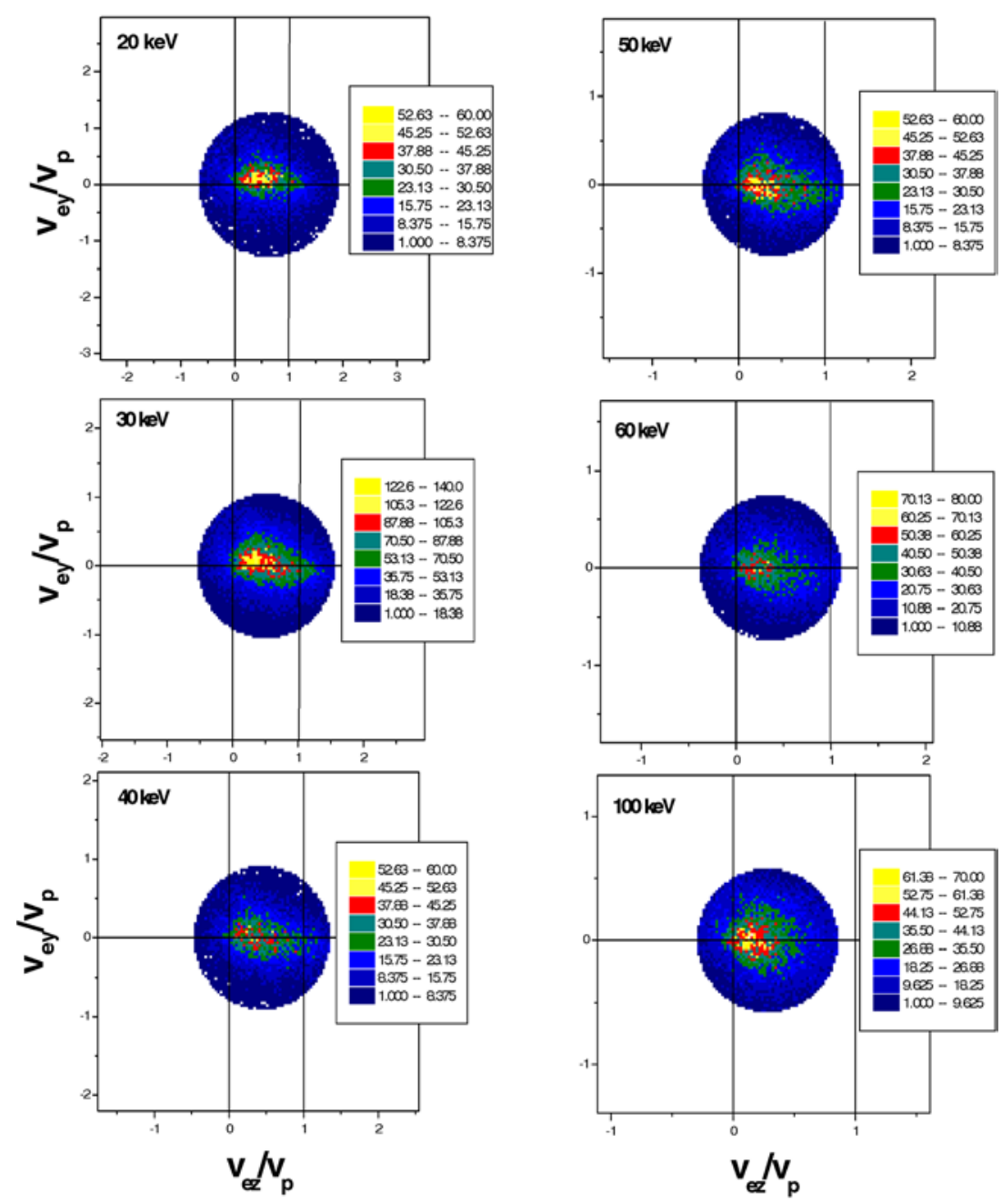

Figure 1. Density plots of the electron velocity spectra projected onto the $x-z$ plane. The recoil momentum is defined to be in the $-y$ direction.

(This figure is in colour only in the electronic version)

is the projectile velocity. Target-centred electron emission would be at the origin and electrons captured to the continuum of the projectile would appear close to the cross at $v_{e} / v_{p}=1$. We see that the forward jet seen by Dörner et al remains visible at $20 \mathrm{keV}$, although the two-fingered structure seen by those authors is not visible at the higher energies. As the projectile velocity increases we see that the electron distribution becomes more and more target centred, as expected.

Figures 2(a) and 3(a) show projections of the data of figure 1 onto the longitudinal and transverse axes, respectively. In the longitudinal projections of figure 2(a), the progression of the distribution from a 'saddle-centred' distribution to a 'target-centred' one is apparent. These data thus document the transition from the molecular-orbital regime to the perturbative regime. The corresponding transverse projections are shown in figure 3(a). The $20 \mathrm{keV}$ transverse momentum distribution is consistent with the $15 \mathrm{keV}$ results of [17]. Both distributions show an asymmetry biased toward the direction of the recoiling target ion. Going to higher impact 
(a)

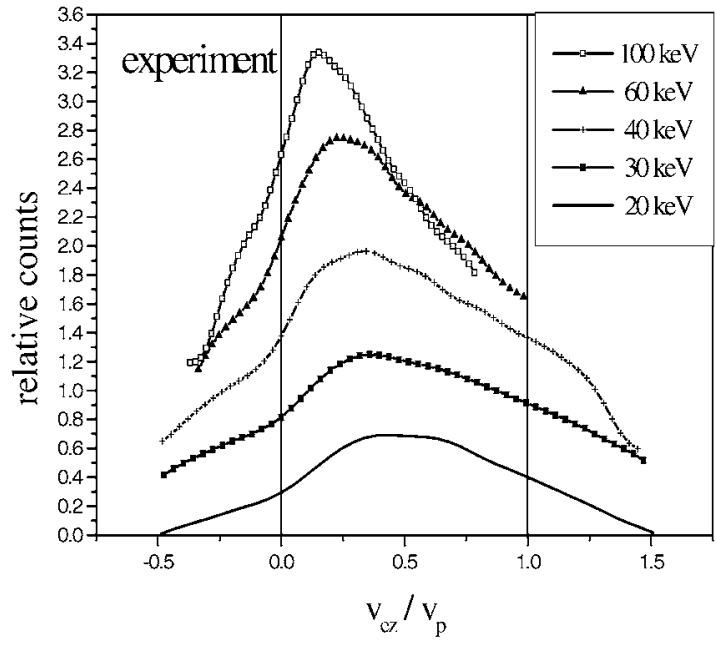

(b)

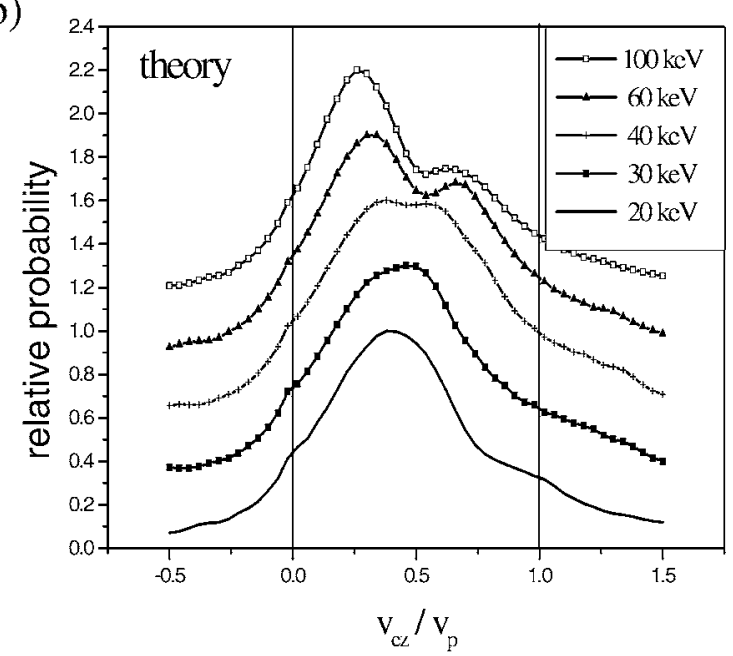

Figure 2. Longitudinal momentum distributions of the ejected electrons. (a) Experiment, (b) theory $(b=1.5 \mathrm{au})$. To guide the eye we have shifted each curve vertically by 0.25 for each increasing energy.

energies, there is perhaps a very weak tendency in the present data for the oscillation to continue, shifting slightly to the opposite side of the recoil at $40 \mathrm{keV}$ and slowly returning to being nearly symmetric at $100 \mathrm{keV}$. We note that the model proposed by Macek and Ovchinnikov [5] would not predict further oscillation of the distribution above $20 \mathrm{keV}$, since the phase difference between $\sigma$ and $\pi$ amplitudes has already become small at this energy and can only approach zero at higher energies.

\section{Theoretical calculation}

In order to provide a theoretical framework for the interpretation of the above results, we have applied the two-centre momentum space discretization (TCMSD) method [8-12] for studying 
(a)

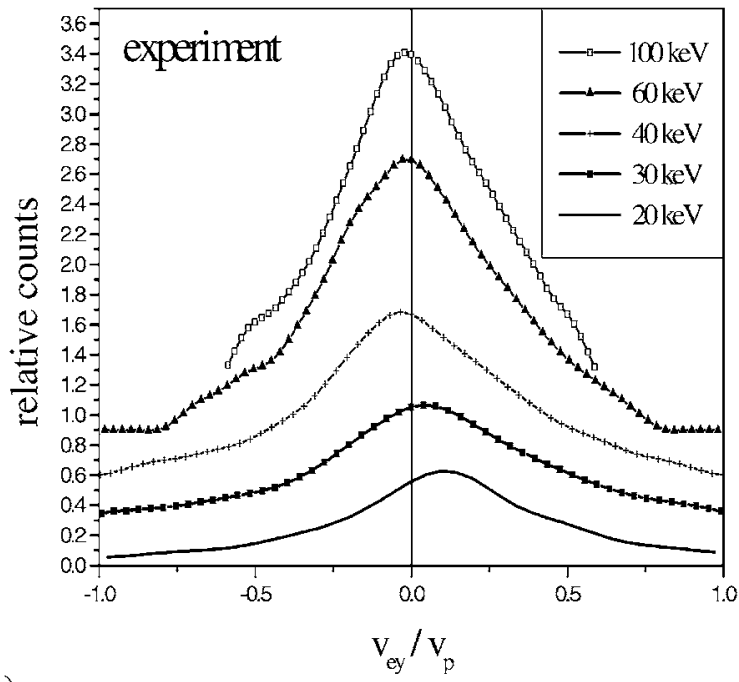

(b)

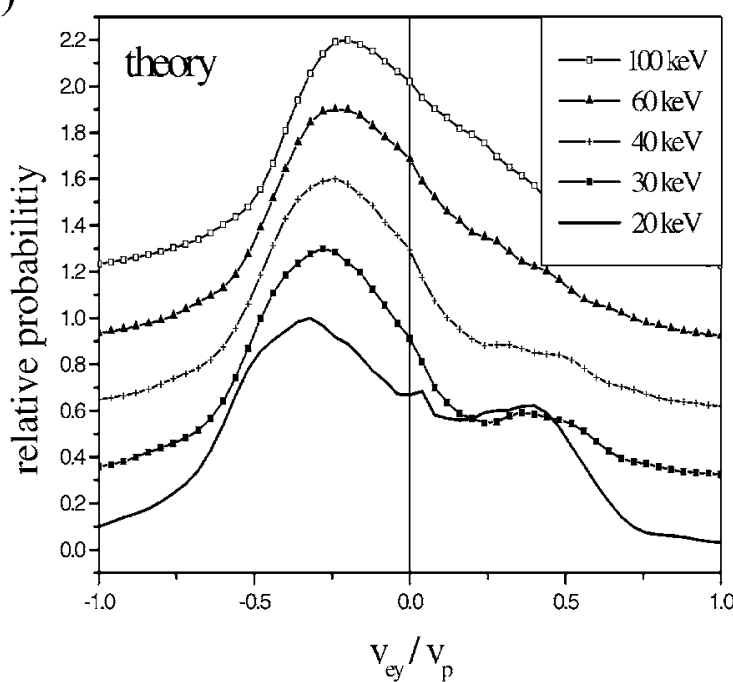

Figure 3. Transverse momentum distributions of the ejected electrons. (a) Experiment (the recoil momentum is defined to be in the $-y$ direction), (b) theory ( $b=1.5 \mathrm{au}$ ). To guide the eye we have shifted each curve vertically by 0.25 for each increasing energy.

the ejected electron momentum distributions in the $\mathrm{p}-\mathrm{He}$ collision system. To account for the He target atom we explored two simple models:

(1) a hydrogenic target with nuclear charge $Z_{T}=1.345$, which gives the correct binding energy of the first electron in the He atom, and

(2) a one-electron spherically symmetric model potential that provides a better fit to excited energy levels in the $\mathrm{He}$ atom and, more importantly, has the correct charge of the $\mathrm{He}^{+}$ion, (see, for example, [20]). 
For the theoretical calculations presented in this paper the two models for the He atom produced very similar results, so we only present theory with the hydrogenic model using the effective charge of 1.345 .

The TCMSD theory gives the wavefunction of the active electron in the form of a twocentre expansion in momentum space:

$$
\Phi(\vec{p}, t)=\Phi_{T}(\vec{p}, t)+\exp \left(\mathrm{i} \vec{R} \cdot \vec{p}-\mathrm{i} p^{2} t / 2\right) \Phi_{P}\left(\vec{p}-\vec{v}_{p}, t\right) .
$$

The target is taken at rest; the projectile velocity is $\vec{v}_{p}$; the internuclear separation is $\vec{R}=\vec{v}_{p} t+\vec{b}$ and $\vec{b}$ is the impact parameter. To obtain the ejected electron momentum distribution from equation (1), we take the projections of the incoherent sum of the target and projectile probability distributions, $\left|\Phi_{T}\right|^{2}+\left|\Phi_{P}\right|^{2}$, since the interference term between the two centres is a rapidly oscillating function of time not observable in the experiment [8].

The TCMSD propagation is not capable, at present, of reaching very large times at which we could compare directly with experimental results, but there is still much that can be learned from the analysis of the momentum space wavefunction at and leading up to the final calculated time of $v t=30$ au $\left(v=v_{p}\right)$. Comparison directly with experiment should also involve an integration of the momentum distributions over impact parameter $\vec{b}$, but for the qualitative discussion that follows one impact parameter is sufficient. We have computed results for impact parameters $b=1.5$ and 2.0 au, which are at the maximum contribution to the total single ionization cross section, and we have found little difference in the behaviour of the ejected electron momentum distribution, so we show results only from the $b=1.5$ calculation.

\section{Comparison of theoretical and experimental results}

For the longitudinal distributions, experimental (figure 2(a)) and theoretical (figure 2(b)) results appear to be in reasonable agreement. Both theory and experiment show a peak near $v_{p} / 2$ for the $20 \mathrm{keV}$ collision. As the impact energy increases, both sets of results show a shift toward the target. The width of the theoretical longitudinal momentum distribution appears to be also consistent with the experiment. The major difference is that the theory shows a small bump near the projectile for the highest impact energies, which is not seen in the experiment. This, however, is not a serious discrepancy when one considers that the theory stops at a finite time and shows results only for one impact parameter. The results for the longitudinal momentum distributions come as no big surprise since it is known that the ejected electron momentum distribution is target centred for high impact energies [24], but the time evolution of the longitudinal momentum distribution, discussed in the next section, shows an interesting evolution towards this expected result.

For the transverse distributions, theoretical results, shown in figure 3(b), do not show the same behaviour as the experiment (figure 3(a)). The theoretical distributions are wider than those of the experiment. Furthermore, the impact-energy dependence is not the same. At $20 \mathrm{keV}$ the transverse momentum distribution of the ejected electron is nearly symmetric, showing a slight tendency to go away from the recoil. At $40 \mathrm{keV}$ and higher, experiment and theory are in qualitative agreement, with both showing asymmetry away from the recoil. Although the experimental and theoretical results appear to disagree, the difference could be coming from the finite ending time of the theoretical calculation, as we discuss below.

\section{Discussion}

Much of the discrepancy between theory and experiment is probably due to the fact that it has not been feasible to carry out the calculation for a $v t$ product beyond about $30 \mathrm{au}$. There is 
evidence that there is considerable evolution of the electron distributions after these times, even for such large distances from the collision region. Figures 4(a) and (b) show the evolution of the transverse momentum distribution of the ejected electron for the 20 and $100 \mathrm{keV}$ collisions, respectively. A feature common to both figures is that they both show a narrowing of the distribution as time progresses. This is expected, since the Coulomb forces from both centres act to slow the electrons ejected transverse to the internuclear separation. An extrapolation of this narrowing could produce a result consistent with the width of the experimental measurements in figure 3(a). Looking at the earliest frame from both collision energies, the distributions are asymmetric, tending to go in a direction opposite the recoil. (The recoil momentum is defined to be in the $-y$ direction.) In fact, if one examines the distributions at approximately equal times, $v t=10$ for the $20 \mathrm{keV}$ collision and $v t=20$ for the $100 \mathrm{keV}$ collision, the transverse momentum distributions look very similar. This points to the possibility that the same mechanism is responsible for the initial ejection of the electron. As time goes on, the saddle potential plays a larger role for the low-energy collision and causes the transverse momentum distribution to evolve toward the direction of the recoiling ion. The plots in figure 4 stop at $v t=30$, so it is not known whether or not the theoretical distribution will eventually move completely to the side of the recoiling ion as the experimental result in figure 3(a) shows.

In figures $4(\mathrm{c})$ and (d) we show the time evolution of the longitudinal momentum distributions corresponding to 20 and $100 \mathrm{keV}$ collisions, respectively. The $20 \mathrm{keV}$ collision at $v t=10$ au shows a distribution, though very broad, centred roughly at the saddle velocity (the saddle velocity is slightly faster than $v / 2$ since the target ion charge is larger than the projectile charge in our model). As $v t$ increases the distribution sharpens slightly and shifts toward the target ion. If saddle point ionization were the only mechanism for ionization, the peak of the longitudinal momentum distribution would remain faster than $v / 2$. Instead the distribution migrates toward the higher target nuclear charge. For the high-energy collision in figure 4(d), longitudinal momentum peaks at the high velocity of $v / 2$ immediately after the collision, $v t=10$. Subsequently, the electrons slow rapidly to the velocity roughly consistent with experiment, figure 2(a). As with the transverse distributions, there is a striking similarity of the longitudinal momentum distributions immediately after the collision at equal times and in unscaled momentum, see $v t=10$ in figure 4(c) and $v t=20$ in $4(\mathrm{~d})$. This again points to the interesting speculation that the mechanism for the initial electron ejection is similar in both collisions.

As discussed in several previous papers, cooling on the ridge is an important link between ionization at finite $v t$ and infinite $v t[11,12]$. The broad aspect ratios predicted by quantal calculations, which extend only to finite $v t$ are now well established. The aspect ratios for CTMC calculations, which do extend to infinite $v t$, are much narrower and closer to the experimental results $[10,11,17]$. It would be quite helpful to have a general procedure, which will analytically continue the results at finite $v t$ to infinity. We note that a preliminary calculation of this type has recently been carried out by Macek et al [21], who used results from a configuration-space lattice calculation by Schultz et al [30], for $\mathrm{p}$ on $\mathrm{H}$. They then analytically propagated these from finite time to infinite time, using a time-dependent harmonic-oscillator propagator. The results showed distinct narrowing of the transverse momentum distributions and the production of electron distributions with an aspect ratio much closer to the experiments for similar systems than has been calculated previously. The results of this calculation and those of this paper both support the conclusion that the major disagreement between theory and experiment in the width of the transverse distributions can be attributed to a long-range transverse 'cooling' of the electrons in the gradually disappearing saddle potential as the collision partners depart. 
(a)

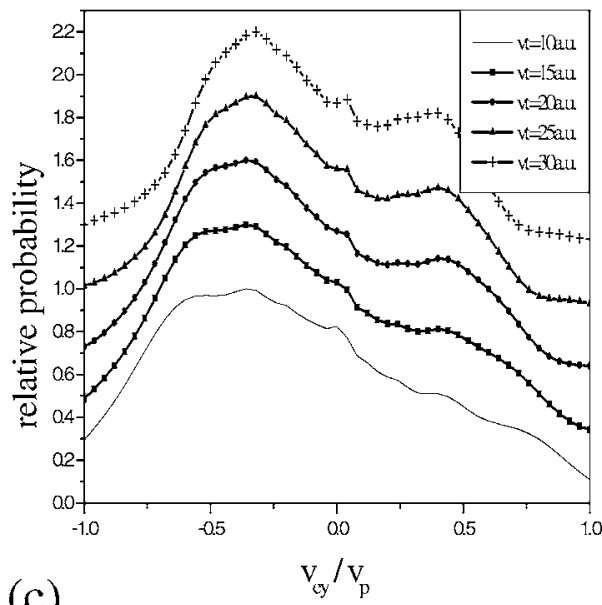

(c)

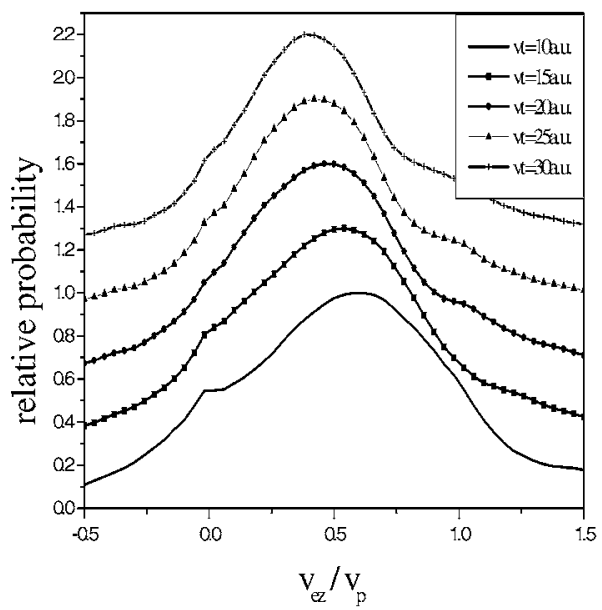

(b)

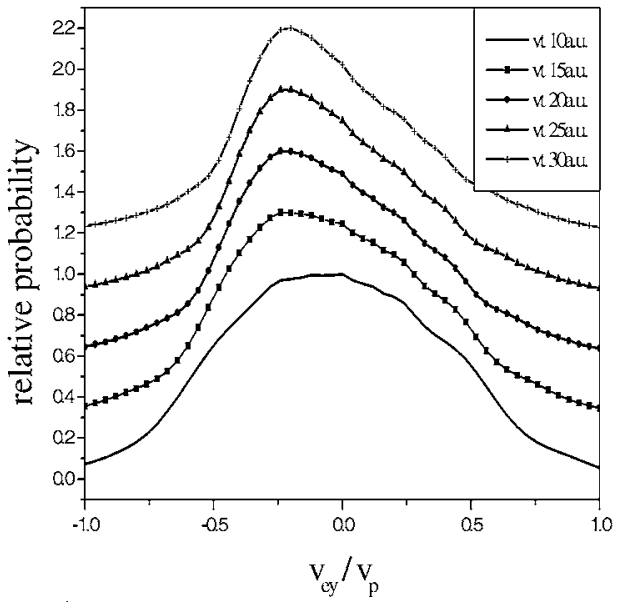

(d)

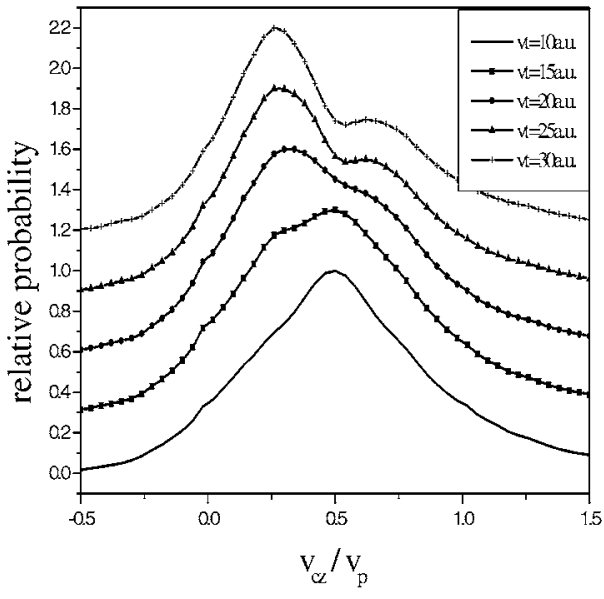

Figure 4. Calculated $v t$ dependence of the momentum distributions for ejected electrons. (a) Transverse momentum at $20 \mathrm{keV}$, (b) transverse momentum at $100 \mathrm{keV}$, (c) longitudinal momentum at $20 \mathrm{keV}$ and (d) longitudinal momentum at $100 \mathrm{keV}$. To guide the eye we have shifted each curve vertically by 0.25 for each increasing energy.

\section{Acknowledgments}

This work was supported by Chemical Sciences, Geosciences and Biosciences Division, Office of Basic Energy Sciences, Office of Science, US Department of Energy. We would like to thank Mike Wells, Al Rankin and Dr Charles Fehrenbach for their technical help in this work.

\section{References}

[1] Olson R E 1983 Phys. Rev. A 271871

[2] Pieksma M and Ovchinnikov S Y 1994 J. Phys. B: At. Mol. Opt. Phys. 274573

[3] Ovchinnikov S Y and Macek J H 1995 Phys. Rev. Lett. 752474 
[4] Ovchinnikov S Y, Macek J H and Khrebtukov D B 1997 Phys. Rev. A 562872

[5] Macek J H and Ovchinnikov S Y 1998 Phys. Rev. Lett. 802298

[6] Winter T G 1988 Phys. Rev. A 374656

[7] Winter T G and Lin C D 1984 Phys. Rev. A 293071

[8] Sidky E Y and Lin C D 1998 J. Phys. B: At. Mol. Opt. Phys. 312949

[9] Sidky E Y and Lin C D 1999 Phys. Rev. A 60377

[10] Sidky E Y, Illescas C and Lin C D 2000 Phys. Rev. Lett. 851634

[11] Sidky E Y, Illescas C and Lin C D 2001 J. Phys. B: At. Mol. Opt. Phys. 34 L163

[12] Sidky E Y and Lin C D 2001 Phys. Rev. A 65012711

[13] Gay T J, Gealy M W and Rudd M E 1990 J. Phys. B: At. Mol. Opt. Phys. 23 L823

[14] Irby V D, Datz S, Dittner P F, Jones N L, Krause H F and Vane 1993 Phys. Rev. A 472957

[15] DuBois R D 1993 Phys. Rev. A 481123

[16] Kravis S D et al 1996 Phys. Rev. A 541394

[17] Dörner R, Khemliche H, Prior M H, Cocke C L, Gary J A, Olson R E, Mergel V, Ullrich J and Schimdt-Böcking H 1996 Phys. Rev. Lett. 774520

[18] Abdallah M, Kravis S, Cocke C L, Wang Y, Rodriguez V D and Stockli M 1997 Phys. Rev. A 562000

[19] Abdallah M A, Cocke C L, Wolff W, Wolf H, Kravis S D, Stockli M and Kamber E 1998 Phys. Rev. Lett. 81 3627

[20] Abdallah M A, Wolff W, Wolf H E, Cocke C L and Stockli M 1998 Phys. Rev. A 58 R3379

[21] Macek $\mathrm{J} 2002$ private communication

[22] Abdallah M A, Cocke C L, Wolff W, Wolf H E and Stockli M 2001 Phys. Rev. A 63024702

[23] Stolterfoht N, DuBois R D and Rivarola R D 1997 Electron Emission In Heavy Ion-Atom Collisions (Springer Series On Atoms And Plasmas) (Berlin: Springer)

[24] Moshammer R, Ullrich J, Kollmus H, Schmitt W, Unverzagt M, Schimdt-Böcking H, Wood C J and Olson R E 1997 Phys. Rev. A 561351

[25] Abdallah M A et al 2000 Proc. 18th Int. Conf. on X-Ray and Inner-Shell Processes (Chicago, IL, 23-27 August 1999) ed R W Dunford et al CP506

[26] Abdallah M A, Landers A, Singh M, Wolff W, Wolf H E, Kamber E Y, Stockli M and Cocke C L 1999 Nucl. Instrum. Methods Phys. Res. B 15473

[27] Weber The al 2001 Phys. Rev. Lett. 86224

[28] Fainstein P D 1999 Phys. Rev. A 60 R741

[29] Fainstein P D and Rodriguez V D 2000 J. Phys. B: At. Mol. Opt. Phys. 334637

[30] Schultz D R, Strayer M R and Wells J C 1999 Phys. Rev. Lett. 823976 